\title{
Composición Química del Aceite de Almendras producidas por el Árbol Olleto (Lecythis minor DC)
}

\author{
Jennifer J. Lafont ${ }^{*}$, Elder A. Calle y Luis C. Durango \\ Universidad de Córdoba, Departamento de Química, laboratorio de Cinética y biocombustibles, \\ Cra 6 No 76-103. Montería-Colombia. (e-mail: jenniferlafontmendoza@gmail.com; e.c.o.12@hotmail.com; \\ luisdurango_87@hotmail.com)
}

* Autor a quien debe dirigirse la correspondencia

Recibido May. 22, 2012; Aceptado Jul. 09, 2012; Versión final recibida Ago. 27, 2012

\section{Resumen}

Se comparan dos métodos de extracción del aceite a partir de las almendras del árbol conocido en el norte de Colombia con el nombre de olleto (Lecythis minor DC). Se analizó su composición química y se determinaron algunas propiedades fisicoquímicas. También se realizó el análisis proximal a la torta y la harina y se determinó la presencia de metales en la almendra. Para extraer el aceite, las almendras fueron trituradas y sometidas a procesos de prensado y extracción con solvente. La composición química del aceite se analizó mediante cromatografía de gases acoplada a masas y los análisis de metales se realizaron con un espectrofotómetro de absorción atómica. Se encontró que el método de extracción más eficiente fue el de solvente. La composición química de la almendra, torta, harina y aceite, indicaron alto porcentaje en carbohidratos, proteína, fibra y minerales, importantes nutrientes que pueden ser empleados en la alimentación humana y animal.

Palabras claves: Lecythis minor DC, almendras, aceite, análisis proximal, metales.

\section{Chemical Composition of Almond Oil obtained from Olleto Trees (Lecythis minor DC)}

\begin{abstract}
Two methods of oil extraction from almonds produced by a tree known in the north region of Colombia as olleto (Lecythis minor DC) are compared. The chemical composition was analyzed and some physicochemical properties were determined. Also, proximal analysis of the cake and the flour was done and the presence of metals in the almond was determined. To extract the oil, the almonds were crushed and subjected to mechanical compression and to solvent extraction. The chemical composition of the oil was analyzed through gas chromatography coupled to mass and the analysis of metals was done with an atomic absorption spectrophotometer. It was found that the most efficient method was solvent extraction. The chemical composition of the almond, cake, flour and oil indicated a high level of carbohydrates, proteins, fiber and minerals, important nutrients that could be used for human and animal consumption.
\end{abstract}

Keywords: Lecythis minor DC, almonds, oil proximal analysis, metals. 


\section{INTRODUCCIÓN}

El árbol de Lecythis minor DC, se conoce en la costa norte de Colombia con los nombres comunes de olleto y olla de mono; crece en zonas tropicales, puede alcanzar hasta $25 \mathrm{~m}$ de altura, su corteza es de color gris cubierto de estrías, el fruto es leñoso con forma de urna unida a una tapa que suelta en la madurez, puede contener de 10 a 16 semillas por fruto, las cuales tienen forma ovoide y color marrón ubicadas en un cuerpo carnoso; el tronco presenta excelentes condiciones para la extracción de madera y es usado en construcciones pesadas; el aceite extraído de las semillas es considerado popularmente como un agente hemostático (López y Montero, 2005).

Existen alrededor de 27 especies del gènero lecythis reseñadas desde Nicaragua hasta Brasil, las mas comunes son: Lecythis ampla Mier, elliptica, minor, ollaria, pisonis, usitata, zabucajo, prancei, serrata, lanceolata, chartacea, mesophyla, tuyrana, corrugata, entre otras (López y Montero, 2005); en la literatura se encuentran pocos estudios de este género los cuales estan enfocados principalmente en la especie Lecythis ollaria por su captaciòn de selenio (Ferri et al., 2004), siendo este elemento una traza fundamental en la dieta diaria la cual varìa entre 50 y $75 \mu \mathrm{g} / \mathrm{dia}$ (Ferguson et al., 2012) pero en grandes concentraciones en el organismo causa intoxicaciòn (Müller y Desel, 2010); por lo cual se analizaron diferentes partes de esta planta para encontrar la región de mayor acumulacion de selenio; el estudio reportó mayor concentración en las semillas, el cual esta ligado al grado de maduración (Lemire et al., 2010); otros estudios realizados a la especie Lecythis ollaria están direccionados hacia el aprovechamiento del selenio contenido en las hojas, en análisis para el tratamiento de cáncer; el selenio parece prevenir varios tipos de cáncer, hasta el momento estos resultados preliminares se han reportado como una ayuda terapéutica potencial a la quimioterapia (Kolachi et al., 2010).

La especie Lecithys pisonis también ha sido estudiada y es sugerida como fuente nutricional por su alta concentración en proteínas, aminoácidos esenciales, ácidos grasos y minerales (Denadai et al., 2007). La composición química de la especie Lecythis usitata fue analizada y se encontró ácidos grasos, tocoferoles, esteroles, elementos inorgánicos, entre otros (Andrade et al., 1999). En la actualidad no se han reportado estudios científicos acerca de la composición química del aceite contenido en las almendras de la especie Lecythis minor DC, motivo por el cual se llevó a cabo este trabajo, donde se evaluó la extracción del aceite por los métodos de prensado y de solvente (soxhlet), su composición y algunas propiedades químicas de los aceites, así mismo se realizó el análisis proximal de la torta residual de la extracción por prensado y de la harina obtenida en la extracción con solvente; además se examinó el contenido de minerales presentes en la almendra, con el fin de encontrar un potencial valor comercial o posible aplicabilidad.

\section{METODOLOGÍA}

Las semillas del fruto del árbol de olleto (Lecythis minor DC), fueron recolectadas en el municipio de Lorica ubicado en el departamento de Córdoba - Colombia, lugar donde abunda esta especie, se tomaron muestras aleatorias en tres lugares (centro, noroeste y sur) de este municipio las cuales se almacenaron en bolsas separadas; luego se seleccionaron de cada grupo las semillas maduras que estuvieran en buen estado sanitario, eliminando las dañadas; posteriormente fueron secadas al sol durante 72 horas para facilitar la separación manual de las almendras. En cada grupo las almendras fueron trituradas utilizando un mortero cerámico, hasta formar una masa fina, las cuales fueron empacadas en bolsas de poliestireno con cierre hermético, numeradas (1, 2 y 3 respectivamente) y almacenadas en la oscuridad a temperatura del laboratorio $\left(22^{\circ} \mathrm{C}\right)$, las cuales se les realizaron la extracción del aceite por los métodos de prensado mecánico y por solvente.

En el primer método, se pesaron $50 \mathrm{~g}$ de la muestra 1 en una balanza analítica OHAUS de $\pm 1 \times 10^{-4} \mathrm{~g}$ de precisión; las almendras trituradas fueron calentadas a $35^{\circ} \mathrm{C}$ y prensadas utilizando una prensa a escala de laboratorio (65 $\mathrm{cm}$ de altura y $23 \mathrm{~cm}$ de ancho), provista de un cilindro con superficie lisa, donde se introduce la muestra y un pistón que es manejado manualmente con un gato hidráulico tipo botella, marca TUV, con alturas: mínima de $18 \mathrm{~cm}$, máxima de $24 \mathrm{~cm}$, elevación de $6 \mathrm{~cm}$ y capacidad máxima de carga de $4000 \mathrm{Kg}$ (4 Ton), el aceite extraído fue recolectado en un beacker y la torta residual se volvió a prensar en las mismas condiciones con el fin de obtener mayor cantidad de aceite, el cual fue almacenado en el mismo recipiente y rotulado (primera y segunda prensada); de igual forma la torta residual de la extracción por prensado fue utilizada para realizar el análisis proximal; este procedimiento se repitió para las muestras 2 y 3 (una vez por cada muestra), para evaluar el valor promedio la desviación estándar $\overline{(X} \pm \sigma) \%$ y calcular el rendimiento, dividiendo la masa total del aceite entre la cantidad de muestra de almendra utilizada. La extracción por solvente se realizó mediante un proceso de percolación-inmersión en un equipo de soxhlet compuesto por un balón de fondo plano, una columna y un condensador, siguiendo la metodología descrita por (Lafont et al., 2011), para el cual se tomó $50 \mathrm{~g}$ de la muestra 1 y se introdujo en un cartucho de papel 
filtro cuya superficie porosa permite al solvente entrar y salir reteniendo a la muestra sólida, esta fue ubicada en la columna del equipo que posee un brazo en forma de sifón, la cual estaba conectada por encima a un condensador y por debajo con un balón de fondo plano que contenía 300mL del solvente hexano (Merck; 99.0\%), este sistema fue colocado en una plancha de calentamiento Schoff manteniendo la temperatura a $70^{\circ} \mathrm{C}$ (cercana al punto de ebullición del hexano $69^{\circ} \mathrm{C}$ ); proceso en el cual los vapores del solvente ascendían hasta el condensador y luego el líquido iba cayendo a la columna donde se encontraba la muestra hasta llenarla por completo, ésta a su vez se descargaba por efecto del sifonado al balón de fondo plano, este proceso o ciclo se repitió automáticamente hasta alcanzar un total de 60 ciclos para cada extracción, correspondiente a un tiempo aproximado de 4.5 horas de reflujo; luego la mezcla obtenida de aceite más solvente fue rotoevaporada, recuperando el solvente y obteniendo el aceite. Este procedimiento se repitió para las muestras 2 y 3 con el hexano; (una vez por cada muestra rotulada 1, 2 y 3) con el propósito de evaluar el valor promedio y la desviación estándar $\overline{(X} \pm \sigma) \%$ para cada análisis; la harina obtenida como subproducto de la extracción por solvente fue utilizada para realizar el análisis proximal.

Ambos aceites obtenidos por prensado y por solventes fueron sometidos a un proceso de desgomado en forma independiente, en el cual se calentaron a $90^{\circ} \mathrm{C}$ y se les adicionó el $10 \%$ de agua destilada con base al volumen del aceite, se dejaron reposar por 30 minutos y fueron centrifugados, para retirar las gomas, fosfátidos y lecitinas presentes (Castro et al., 2007). A los aceites desgomados (obtenidos por prensado y solvente), se les realizaron los análisis de: humedad y materia volátil, corrosión en lámina de cobre, índices de acidez, yodo, peróxido y saponificación; para cada análisis fue utilizada una muestra de $5 \mathrm{~g}$. Al aceite obtenido por prensado además se le realizó la identificación de los compuestos químicos presentes en el aceite; no se utilizó el de solvente para evitar que este interfiriera de alguna forma en la identificación de los componentes. Para determinar el contenido de humedad (Lafont et al., 2011) se tomó un crisol limpio, seco $\left(105^{\circ} \mathrm{C}\right.$ por 1 hora), se pesó $5 \mathrm{gr}$ de la muestra $\left(\mathrm{W}_{\mathrm{M}}\right)$, la cual fue introducida en una mufla E\&Q dotada con una termocoupla $\mathrm{K}$, durante 4 horas a la temperatura de $105^{\circ} \mathrm{C}$; posteriormente fue retirada y pesada $\left(\mathrm{W}_{\mathrm{i}}\right)$, luego se colocó en un desecador y se pesó hasta un valor constante $\left(W_{f}\right)$, se calculó el contenido de materia seca mediante la ecuación 1 , donde MS representa el porcentaje de materia seca, $W_{i}$ es el peso inicial del crisol con la muestra húmeda, $W_{f}$ es el peso final del crisol con la muestra seca, $W_{M}$ es el peso de la muestra. El porcentaje de humedad $(\mathrm{H})$ se calculó de la diferencia de 100 con el contenido de matera seca (MS).

$M S=\left[\left(\frac{W_{f}-W_{i}}{W_{M}}\right)\right] * 100 \%$

Para hacer el análisis de corrosión en lámina de cobre (ASTM D130 -10; 2003) se tomó la muestra y se le adicionó una lámina de cobre pulida, luego se calentó a $50^{\circ} \mathrm{C}$ durante 180 minutos, posteriormente fue retirada y lavada con metanol, con el fin de comparar el color obtenido en la lámina con el estándar de la norma. Para realizar el índice de acidez, se pesó $5 \mathrm{~g}$ de aceite y se adicionó $50 \mathrm{~mL}$ de la solución etanol-éter dietílico en proporción 1:1 a temperatura ambiente, luego se agregaron 3 gotas de fenolftaleína como indicador ácido-base y se tituló con hidróxido de potasio hasta el viraje de color; en esta prueba se expresan los ácidos grasos libres como ácido oleico (Hernández et al., 2007). El índice de yodo se realizó pesando la muestra y disolviéndola en tetracloruro de carbono, luego se le agregó $25 \mathrm{~mL}$ del reactivo de Wijs y se dejó reposar la solución en la oscuridad por $30 \mathrm{~min}$, posteriormente fue agregada una solución de yoduro de potasio al $15 \%$ y se tituló con tiosulfato de sodio $0.1 \mathrm{~N}$ utilizando almidón como indicador hasta que el color azul desapareció (Hernández, 2007). Para el índice de peróxido, se pesó la muestra y fue vertida en una solución de ácido acético-cloroformo 3:2; se agregó yoduro de potasio saturado y agua, posteriormente se tituló con tiosulfato de sodio $0.01 \mathrm{~N}$ utilizando almidón como indicador hasta que el color amarillo desapareció (AOCS Cd 8-53; 2003).

El índice de saponificación se realizó tomando un erlenmeyer de $250 \mathrm{~mL}$ en el cual se pesó $5 \mathrm{~g}$ de aceite, se le agregó $25 \mathrm{~mL}$ de solución etanólica de hidróxido de potasio $0.5 \mathrm{~N}$ y fue sometido a reflujo durante 60 min, posteriormente se agregó $1 \mathrm{~mL}$ de solución alcohólica de fenolftaleína al 1\% y se tituló con ácido clorhídrico $0.5 \mathrm{~N}$; también se realizó el blanco con las mismas condiciones (AOCS Cd 3-25; 2003). Los ensayos de las muestras y del blanco se realizaron por triplicado. El análisis de la composición química del aceite extraído por prensado se realizó utilizando un cromatógrafo de gases, Agilent 6890N con detector selectivo de masas Agilent 5973N y Software - MSD Productivity Chemstation, con columna capilar Zebron ZB-5 (Phenomenex) de $30 \mathrm{~m} \times 0.25 \mathrm{~mm}$ I.D, $0.25 \mu \mathrm{m}$ de espesor de película, detector FID, sistema de inyección split, las condiciones del método utilizado fueron rampa de calentamiento de $15^{\circ} \mathrm{C} / \mathrm{min}$ hasta $300^{\circ} \mathrm{C}$, temperatura máxima de la columna $325 \mathrm{C}$, temperatura del inyector y del detector $310^{\circ} \mathrm{C}$ tiempo total de corrida igual a 60min, utilizando el helio como gas de arrastre. El procedimiento empleado para la cuantificación de los compuestos orgánicos presentes en el aceite fue el de inyección directa, previa dilución 
con isopropanol como solvente para volatilizar las muestras y facilitar su paso a través de la columna del equipo, por lo cual no fue necesario metilarlas. Para preparar las muestras (ASTM D-6584, 2003) se utilizó un vial para cromatografía marca AGILENT con volumen de $2 \mathrm{~mL}$, se le agregaron $50 \mu \mathrm{L}$ de aceite, $1 \mu \mathrm{L}$ de estándar interno (n-tetradecano) y se agregó isopropanol hasta aforar. De esta solución se tomó $1 \mu \mathrm{L}$ para ser inyectada en el cromatògrafo de gases acoplado a masas; con la ayuda de una jeringa Agilent con capacidad de 0 -10 $\mu \mathrm{L}$; este ensayo se realizó por duplicado, es de anotar que antes de cada inyección se purgó la jeringa 10 veces con el solvente y 10 con la muestra. La identificación se realizó por comparación de los espectros de masas y los tiempos de retención de los compuestos identificados en el aceite con las bases de datos NIST, WILEY Y ADAMS, que posee el equipo, con una correspondencia del 98\%. Tanto a la torta residual de la extracción por prensado, como a la harina obtenida por la extracción con solventes, se les realizaron los análisis de humedad y materia volátil, cenizas, proteínas, grasa cruda, fibra y carbohidratos; todos los ensayos fueron realizados por triplicado. Para determinar el contenido de humedad se siguió el procedimiento descrito anteriormente (Lafont et al., 2011). El contenido de cenizas (C) (Alvis et al., 2008) se determinó pesando $2 g$ de muestra $\left(W_{M}\right)$ en un crisol seco, el cual fue colocado en una mufla durante 2 horas a la temperatura de $100^{\circ} \mathrm{C}$, después se pesó $\left(\mathrm{W}_{\mathrm{i}}\right)$ y se calcinó a $550^{\circ} \mathrm{C}$ hasta peso constante (6 horas); las cenizas obtenidas se pesaron $\left(\mathrm{W}_{\mathrm{f}}\right)$ y se calculó su porcentaje usando la ecuación 2.

$C=\left[\left(\frac{W_{f}-W_{i}}{W_{M}}\right)\right] * 100 \%$

El contenido de fibra total (F) (Alvis et al., 2008) se determinó mezclando $2 \mathrm{~g}$ de la muestra $\left(\mathrm{W}_{\mathrm{M}}\right) \mathrm{con} 200 \mathrm{~mL}$ de ácido sulfúrico al 1.25\% v/v para realizar la hidrolisis ácida y luego se calentó por 30 minutos hasta ebullición, pasado este tiempo se filtró y el residuo se lavó con agua caliente varias veces, hasta obtener el agua de lavado libre de ácido, luego fue vertido en un beacker y se adicionó 200mL de solución caliente de hidróxido de sodio al $1.25 \%$ p/v, se dejó hervir por 30min, después fue filtrado y lavado varias veces con agua hirviendo y con pequeñas cantidades de alcohol, el residuo es secado a $105^{\circ} \mathrm{C}$ durante 2 horas, pesado y registrado como peso inicial $\left(\mathrm{W}_{\mathrm{i}}\right)$; finalmente fue llevado a la mufla durante 1 hora a la temperatura de $500^{\circ} \mathrm{C}$, pesado y registrado como peso final $\left(\mathrm{W}_{\mathrm{f}}\right)$; el porcentaje de fibra se determinó mediante la ecuación 3.

$F=\left[\left(\frac{W_{f}-W_{i}}{W_{M}}\right)\right] * 100 \%$

El contenido de proteínas (P) (Alvis et al., 2008) se determinó por el método Kjeldahl; mediante la digestión obtenida al mezclar $2 \mathrm{~g}$ de la muestra con $10 \mathrm{~mL}$ de ácido sulfúrico concentrado y $5 \mathrm{~mL}$ de la solución digestora o catalizador (3,5 g de sulfato de potasio anhidro; 1,0 g de sulfato de cobre pentahidratado y $15 \mathrm{~mL}$ de ácido sulfúrico concentrado al 98\%), luego el sistema se sometió a calentamiento por 1.5 horas; posteriormente, se dejó reposar y se adicionaron en forma lenta (por un tiempo de $4 \mathrm{~min}$ ) 30mL de agua y $80 \mathrm{~mL}$ de $\mathrm{NaOH}$ al $40 \% \mathrm{p} / \mathrm{v}$, finalmente la mezcla fue destilada dejando el terminal del equipo de destilación inmerso en $30 \mathrm{~mL}$ de una solución de ácido bórico al 4\%v/v y una gotas de indicador azul de bromotimol hasta observar el cambio de color de amarillo a azul, hasta obtener un volumen de 60mL (ácido bórico más destilado); el cual fue titulado con $\mathrm{HCl} 0.25 \mathrm{~N}$. El porcentaje de proteína $\mathrm{P}$ se determinó mediante la ecuación 4; donde $\mathrm{V}$ es el volumen en $\mathrm{mL}$ de ácido gastado en la titulación, $\mathrm{N}$ representa la normalidad del ácido clorhídrico, 14 son los equivalentes-gramo del nitrógeno, $f$ es el factor proteico en este caso es de 6.25 y $\mathrm{W}$ es el peso de la muestra en gramos.

$P=\left[\frac{(V * N * 14 f)}{\left(100 * W_{M}\right)}\right] * 100 \%$

Para determinar el contenido de grasa cruda (Alvis et al., 2008), se pesó 1 gr de muestra y se colocó en un dedal de papel filtro para extracción por soxhlet; luego sobre un crisol seco y tarado se vertieron $70 \mathrm{~mL}$ de éter de petróleo y se empezó la extracción de la muestra durante una hora, posteriormente se evaporó el solvente y el crisol con la grasa extraída se colocó en un desecador hasta peso constante; el porcentaje de grasa cruda $\left(G_{c}\right)$ se calculó utilizando la ecuación 5 , donde $W_{i}$ es el peso inicial del crisol vacío, $W_{f}$ es el peso final del crisol después del tratamiento y $\mathrm{W}_{\mathrm{M}}$ es el peso de la muestra.

$G c=\left[\left(\frac{W_{f}-W_{i}}{W_{M}}\right)\right] * 100 \%$ 
El porcentaje de carbohidratos se calculó mediante la diferencia de $100 \%$ con la sumatoria de los porcentajes de proteína cruda, lípidos, fibra y cenizas (Alvis et al., 2008) respectivamente para cada muestra analizada. Los análisis de metales se realizaron utilizando un espectrofotómetro de absorción atómica marca Thermo Electron Corporation Modelo: S4AA System serie 6E711397. En este análisis se calcinó $2 \mathrm{~g}$ de la almendra para obtener las cenizas, utilizando el procedimiento descrito anteriormente, estas fueron disueltas en un balón volumétrico donde se les adicionó $3 \mathrm{~mL}$ de ácido clorhídrico y agua hasta alcanzar un volumen de $25 \mathrm{~mL}$; con el fin de disolver los minerales presentes en ella, esta solución fue filtrada y empleada para analizar el contenido de metales en el equipo (Amin et al., 2007). El límite de cuantificación (LC) del método estuvo en el rango de (0.02-0.03) $\mathrm{mg} / \mathrm{L}$, la exactitud (R) entre (110 y 91.0) \% y la precisión (RSD) en el rango de (1.52-6.83) \%. El control de calidad analítica se realizó utilizando el estándar certificado titrisol-Merck (1000mg); mediante su lectura para verificar que las mediciones estuvieran dentro del rango establecido.

\section{RESULTADOS Y DISCUSIÓN}

El aceite de las almendras de Olleto obtenido por ambos mètodos (prensado y solvente) es de color amarillo brillante, poco viscoso y con olor a nuez, en general se puede decir que presenta buena apariencia. En la tabla 1, se muestra el valor promedio del rendimiento y la desviación estándar en porcentaje $\overline{(X} \pm \sigma) \%$ para la extracción del aceite por los mètodos de prensado y de solvente; es evidente que el valor de 71.99 ( \pm 0.25)\% obtenido por prensado presenta un rendimiento mucho menor que $93.71( \pm 0.39) \%$ obtenido en la extracciòn con el sovente hexano; siendo este el mètodo mas eficiente, lo cual probablemente puede ser debido a que el hexano es un hidrocarburo que no posee grupos funcionales en su estructura quìmica y cuya cadena carbonada es similar a la de los compuestos que forman el aceite; lo cual facilita la liberacion de las molèculas oleaginosas de la nuez durante todo el proceso de extracciòn. El método de prensado muestra una eficiencia menor comparado con la extracciòn por solvente, esto puede ser debido a que en la torta quedan retenidas molèculas de aceite y en consecuencia este porcentaje es menor; sin embargo se puede resaltar que ambos resultados son buenos para llevar a cabo esta extraccion a nivel industrial.

Tabla1: Porcentajes de aceite extraídos de la almendra de Olleto por ambos métodos

\begin{tabular}{|l|c|c|c|}
\hline Método & & Solvente & Rendimiento $\overline{(X \pm \sigma) \%}$ \\
\hline Prensado & & & $71.99 \pm 0.25$ \\
\hline \multicolumn{2}{|l|}{ Solvente con inmersión -percolación } & Hexano & $93.71 \pm 0.39$ \\
\hline
\end{tabular}

En la tabla 2, se reportan los valores promedios del anàlisis proximal y la desviaciòn estàndar en porcentaje de la torta $\overline{(X} \pm \sigma) \%$ y de la harina obtenidas posteriormente a los procesos de extracciòn con prensado y solvente respectivamente; es evidente la presencia de un alto porcentaje de carbohidratos presente en la torta $(86.191 \pm 0.26) \%$ y aún más elevada en la harina $(89.033 \pm 0.52) \%$, lo cual representa un importante valor nutritivo en alimento para animales, por ser la fuente de energía más importante en los rumiantes, ya que estos se transforman en azucares y grasas, los carbohidratos son aldehídos o cetonas polihidroxilados que se encuentran en los alimentos en forma de monosacáridos como la glucosa y la fructosa, oligosacáridos como la sacarosa y lactosa y polisacáridos como la celulosa y el almidón. El contenido de cenizas representa los elementos minerales presentes, los cuales pueden clasificarse como macro elementos y micro elementos; los valores de cenizas para la torta $(5.185 \pm 0.52) \%$ y para la harina (4.967 \pm $0.97) \%$ son muy similares, lo cual indica que no es afectado significativamente por el método empleado en la extracción del aceite. Los valores de fibra indican la presencia de carbohidratos de difícil digestión como la celulosa, hemicelulosa, pentosanos, lignina entre otros; encontrándose en mayor proporción en la torta $(2.843 \pm 0.76)$ con relación a la harina $(2.184 \pm 0.24) \%$, respectivamente.

Tabla 2: Análisis proximal de la torta y harina de la almendra del Olleto

\begin{tabular}{|l|c|c|}
\hline Componente & Torta $\overline{(X} \pm \sigma) \%$ & Harina $\overline{(X} \pm \sigma) \%$ \\
\hline Humedad & $0.306 \pm 0.32$ & $0.813 \pm 0.18$ \\
\hline Grasa cruda & $0.543 \pm 0.41$ & $0.086 \pm 0.74$ \\
\hline Proteínas & $4.250 \pm 0.11$ & $2.513 \pm 3.21$ \\
\hline Cenizas & $5.185 \pm 0.52$ & $4.967 \pm 0.97$ \\
\hline Carbohidratos & $86.191 \pm 0.26$ & $89.033 \pm 0.52$ \\
\hline Fibra total & $2.843 \pm 0.76$ & $2.184 \pm 0.24$ \\
\hline
\end{tabular}


El contenido de proteínas presente en la torta $(4.250 \pm 0.11) \%$ fue 1.7 veces mayor que el obtenido en la harina $(2.513 \pm 3.21) \%$, en este caso si influyó el método de extracción del aceite, siendo el de prensado más favorable para obtener mejor rendimiento; las proteínas también son importantes en los alimentos de animales porque están formadas por cadenas de aminoácidos unidos por enlaces peptìdicos los cuales participan activamente en diferentes funciones vitales del organismo como el metabolismo, la respuesta inmunológica y la contracción muscular. El porcentaje de grasa cruda encontrada en la torta $(0.543 \pm 0.41) \%$ fue seis veces mayor que la encontrada en la harina $(0.086 \pm 0.74) \%$, lo cual sugiere que el método de extracción por prensado extrajo menor cantidad de aceite y por lo tanto retuvo mayor cantidad de grasa en la torta, por el contrario en la harina se encontró muy poca cantidad de grasa, lo cual corrobora que el método de extracción por solvente es el mejor para extraer aceite. El porcentaje de humedad en la torta $(0.306 \pm 0.32) \%$ fue menor que la obtenida en la harina $(0.813 \pm 0.18) \%$, posiblemente este aumento se debe a trazas de solvente que no pudieron ser totalmente retirados en el proceso de rotoevaporación. En la tabla 3 se reportan los valores promedio de minerales presentes en las cenizas de la almendra del olleto y su desviaciòn estàndar en microgramos por gramo de muestra $\overline{(X} \pm \sigma) \mu \mathrm{g} / \mathrm{g}$; de todos los minerales presentes se hallaron en mayor proporciòn (macronutrientes), el potasio (10150.50 \pm 0.37 ) $\mu \mathrm{g} / \mathrm{g}$ le sigue el fòsforo $(6750.90 \pm 0.24) \mu \mathrm{g} / \mathrm{g}$, magnesio $(2620.93 \pm 0.10) \mu \mathrm{g} / \mathrm{g}$, calcio $(1280.66 \pm 0.48) \mu \mathrm{g} / \mathrm{g}$ y bario $(987.16$ $\pm 4.10) \mu \mathrm{g} / \mathrm{g}$, en menor cantidad (micronutrientes) se encontrò el sodio (91.13 \pm 1.14$) \mu \mathrm{g} / \mathrm{g}$, hierro (42.04 \pm $0.21) \mu g / g$, zinc $(38.25 \pm 0.85) \mu g / g$, selenio $(26.44 \pm 0.32) \mu g / g$, manganeso (21.96 \pm 4.10$) \mu g / g$ y cobre $(12.02 \pm 1.14) \mu \mathrm{g} / \mathrm{g}$ estos metales tambien han sido reportados para la Lecythis usitata (Andrade et al; 1999), en proporciones parecidas de mayor a menor y valores comparables, como se describen a continuaciòn, potasio $10300.0 \mu \mathrm{g} / \mathrm{g}$, fòsforo $6690.0 \mu \mathrm{g} / \mathrm{g}$, magnesio $2500.0 \mu \mathrm{g} / \mathrm{g}$, calcio $1240.0 \mu \mathrm{g} / \mathrm{g}$,bario $1000.0 \mu \mathrm{g} / \mathrm{g}$, hierro $45.0 \mu \mathrm{g} / \mathrm{g}$, zinc $35.0 \mu \mathrm{g} / \mathrm{g}$, selenio $28.8 \mu \mathrm{g} / \mathrm{g}$ y manganeso $26.7 \mu \mathrm{g} / \mathrm{g}$. De igual forma en la Lecythis pisonis (Denadai et al., 2007) presenta mayores valores para el potasio, fòsforo, magnesio y calcio, ademas del manganeso, zinc, cobre, hierro y sodio, minerales importantes en la dieta humana. En cuanto al selenio es un micronutriente fundamental para las personas, es un poderoso antioxidante que protege al organismo de toxicos como los metales pesados, incrementa la fertilidad en los hombres al aumentar la producciòn del semen y la motilidad de los espermatozoides; en la dieta de las personas se recomienda ingerir dosis entre 50 y $75 \mu \mathrm{g}$ por dìa, de acuerdo con la edad y el sexo, el promedio para hombres se encuentra entre 70 y $75 \mu \mathrm{g}$ por dìa; (Ferguson et al., 2012) los valores de selenio hallados en la almendra del olleto $(26.44 \pm 0.32) \mu \mathrm{g} / \mathrm{g}$, estan por debajo de este lìmite, razón por el cual este elemento con esta concentraciòn no es considerado lesivo para el organismo, por el contrario podrìa ser aprovechado en la dieta de personas o animales. En las vacas lecheras, la deficiencia de este metal està relacionada con enfermedades como retenciòn de la placenta, infertilidad, mortalidad embrionaria temprana, mastitis clinica entre otros, por ello se recomienda que deben consumir diariamente $0.3 \mathrm{mg}$ Se/ $\mathrm{Kg}$ ò $6 \mathrm{mg}$ de selenio (Gerloff, 1992).

Tabla 3: Minerales presentes en la almendra del Olleto

\begin{tabular}{lc}
\hline Minerales & Torta $\overline{(X} \pm \sigma) \mu g / g$ \\
\hline Potasio & $10150.50 \pm 0.37$ \\
Cobre & $12.02 \pm 1.14$ \\
Sodio & $91.13 \pm 1.14$ \\
Magnesio & $2620.93 \pm 0.10$ \\
Zinc & $38.25 \pm 0.85$ \\
Hierro & $42.04 \pm 0.21$ \\
Fósforo & $6750.90 \pm 0.24$ \\
Calcio & $1280.66 \pm 0.48$ \\
Manganeso & $21.96 \pm 4.10$ \\
Bario & $987.16 \pm 4.10$ \\
Selenio & $26.44 \pm 0.32$ \\
\hline
\end{tabular}

En la tabla 4, se describen las propiedades analizadas para el aceite de la almendra del olleto extraìdo por los mètodos de prensado y soxhlet; estos valores corresponden al porcentaje del valor promedio y la desviación estándar $\overline{(X} \pm \sigma) \%$ para cada anàlisis. El porcentaje de humedad y materia volátil del aceite extraído por prensado $(0.08 \pm 0.16) \%$, se encuentra dentro del intèrvalo internacional (CODEX) establecido para aceites de uso alimenticio (FAO Y OMS, 1999), lo cual pone de manifiesto que el proceso de desgomado no influye en forma significativa en la humedad del aceite, el valor del porcentaje de humedad y materia volátil del aceite extraído por soxhlet $(0.14 \pm 0.35) \%$ es mayor que el de prensado $(0.08 \pm 0.16) \%$ y tambien cumple con la norma, este aumento puede ser debido a trazas del solvente hexano en el aceite que no fueron eliminadas totalmente. 
Tabla 4: Propiedades analizadas para los aceites extraídos por prensado y soxhlet de las almendras del Olleto

\begin{tabular}{|l|c|c|c|}
\hline \multicolumn{1}{|c|}{ Índices } & Prensado $\overline{(X} \pm \sigma) \%$ & Soxhlet $\overline{(X} \pm \sigma) \%$ & CODEX \\
\hline & $\overline{(X} \pm \sigma) \%$ & $\overline{(X} \pm \sigma) \%$ & \\
\hline Humedad (\%) & $0.08 \pm 0.16$ & $0.14 \pm 0.35$ & 0.2 \\
\hline Acidez $(\mathrm{mg} \mathrm{KOH} \mathrm{/} \mathrm{g)}$ & $2.35 \pm 0.12$ & $3.85 \pm 0.03$ & 4.0 \\
\hline Yodo $(\mathrm{cg} \mathrm{I/g)}$ & $86.37 \pm 0.27$ & $85.37 \pm 0.67$ & $75.0-94.0$ \\
\hline Peróxido (meq $\left.\mathrm{O}_{2} / \mathrm{Kg}\right)$ & $1.06 \pm 0.09$ & $1.39 \pm 0.01$ & 15.0 \\
\hline Saponificación $(\mathrm{mg} \mathrm{KOH} / \mathrm{g})$ & $188.14 \pm 0.17$ & $190.02 \pm 0.05$ & $184.0-196.0$ \\
\hline Corrosiòn lamina de cobre & $1 \mathrm{~A}$ & $1 \mathrm{~B}$ & \\
\hline
\end{tabular}

Los valores del índice de acidez del aceite de olleto extraìdo por los mètodos de prensado y solvente se encuentran dentro del valor màximo establecido por la norma internacional (CODEX); esto indica que este aceite puede ser recomendable para uso alimenticio, (FAO Y OMS, 1999); el aceite obtenido por prensado arrojò menor valor de acidez con respecto al de solvente, lo cual indica que es un poco más estable con el tiempo. Los valores del indice de yodo para los aceites extraídos por prensado y por soxhlet estan acorde con el intervalo establecido en la norma internacional (CODEX) para aceites de uso en la industria alimenticia; pero tambien este aceite puede ser clasificado como no secante por presentar valores por debajo de $100 \mathrm{cg} \mathrm{l/g}$, caracterìstica que favorece la utilizaciòn de este aceite en la elaboraciòn de jabones sòlidos y lìquidos, cremas, entre otros productos (Lafont et al., 2011); este índice constituye una medida del grado de insaturación del aceite, característica que se encuentra ligada a propiedades como el punto de fusion, densidad, peso molecular, entre otras.

El índice de peróxido es un indicador del grado de oxidacion o descomposicion (rancidez) del aceite en el momento de la prueba; como se puede observar en la tabla 4, ambos valores se encuentran por debajo del limite maximo permitido por la norma, teniendo en cuenta que se considera que los productos con indices superiores a 2 meq $\mathrm{O}_{2} / \mathrm{Kg}$ son más propensos a mostrar rancidez (Belen et al., 2005), lo cual indica que ambos son bastante estables a la degradacion. Los valores del índice de saponificación para el aceite extraído por prensado y solvente se encuentran dentro del intervalo establecido por la norma CODEX para los aceites de uso en la industria alimenticia. En cuanto al anàlisis de corrosiòn en lamina de cobre para el aceite extraído de las almendras de olleto por el metodo de prensado fue de $1 \mathrm{~A}$, lo cual indica que se encuentra dentro de las normativas establecidas para aceites que pueden ser utilizados como materia prima en la medicina y en la industria. El aceite extraído con solvente (soxhlet) encaja en la categoría 1B, lo que indica que tiene un poder corrosivo un poco mayor al de prensado, esto puede ser debido a la presencia de ácidos grasos libres como producto de la hidrólisis de los triglicéridos causada por la alta humedad que se presenta en los aceites extraídos por este método. De acuerdo con los valores de corrosión obtenidos, se puede decir que ambos aceites tienen un bajo poder de corrosión y por tal motivo pueden ser almacenados en los recipientes que comúnmente se designan para estos fines.

De acuerdo con los análisis espectroscópicos, se lograron identificar 17 compuestos equivalentes al $90.47 \%$ del aceite, presentándose en mayor proporción el escualeno con el (44.64\%), luego el (E,E)-2,4decadienal (10.54\%), el ácido oleico (6.94\%), el hexanal (5.57\%) y el ácido linoléico (4.04\%); correspondientes al $71,73 \%$. El otro $18.74 \%$ restante fue identificado en pequeñas concentraciones de los siguientes compuestos: p-Xileno (2.47\%); 1-metil-4-(5-metil-1-metileno-4-hexenil) Ciclohexeno (2.31\%); Ácido Palmítico (2.26\%); 1-etil-2-metil Ciclopropano (2.04\%); 3-metil-2-butenil-benceno (2.10\%); Hexadecanoato de metilo (1.91\%); (Z)-2-Decenal (1.57\%); 9-Octadecenoato de metilo (1.48\%); Nonanal (0.88\%); 9,17-Octadecadienal (0.76\%); (E) -2-Octenal (0.48\%); E,E)- 9,12-Octadecadienoato de metilo $(0.48 \%)$. Es importante resaltar que el escualeno es el compuesto con mayor proporción en el aceite de olleto (44.64\%), es un triterpeno obtenido con fines comerciales a partir del aceite de hígado del tiburón, es parte natural y esencial de la síntesis del colesterol, las hormonas esteroides y la vitamina $D$ en el cuerpo humano; el escualeno es ampliamente utilizado en la dieta de cerdos para mejorar su reproducción (Zhang et al., 2008); también en cosméticos, como antioxidante, emoliente e hidratante; ha demostrado efecto preventivo a la carcinogénesis, también actúa como adyuvante inmunológico en vacunas contra la gripe y la nueva influenza AH1N1, el herpes, el paludismo, entre otros; (Lippi et al., 2010). La presencia de escualeno en el aceite de olleto hace que esta materia prima adquiera gran importancia para la producción, extracción y uso del escualeno a nivel farmacológico y comercial. Como se puede apreciar el aceite de las almendras de olleto (lecythis minor DC) posee un $13.24 \%$ de ácidos grasos (oleico, linolèico, palmítico), lo cual es importante para los productos cosméticos y en la alimentación, sin embargo es pobre en cantidad y variedad para la producción de biodiesel ya que para ello se requieren aceites ricos en ácidos grasos para 
poder llevar a cabo la reacción de transesterificación, razón por el cual se descarta este uso. Se puede decir que el 32.59\% restante de la composición química del aceite está formado por hidrocarburos, terpenos, esteres y aldehídos de alto peso molecular, además poseen compuestos aromáticos que le proporcionan el olor y el sabor característico al aceite.

\section{CONCLUSIONES}

De los resultados, análisis y discusión se pueden extraer las siguientes conclusiones: 1) el método empleado para la extracción de aceite a partir de almendras del olleto (Lecythis minor DC) que presentó mejor rendimiento, fue el realizado con solvente (hexano); 2) los análisis proximales de la torta y harina mostraron alta concentración en nutrientes importantes para la alimentación humana y animal, así como los minerales encontrados en la almendra, significativos en la nutrición de las personas; 3) las propiedades fisicoquímicas calculadas para los aceites están acorde con los parámetros establecidos por la norma CODEX para la industria alimenticia; 4) La composición química del aceite indicó mayor porcentaje de escualeno y presencia de ácidos grasos, compuestos importantes en la alimentación y reproducción animal.

\section{AGRADECIMIENTOS}

Al laboratorio de Instrumental del Departamento de Química de la Universidad de Córdoba y al laboratorio de Instrumental de la Universidad Nacional -Sede Medellín por el apoyo brindado.

\section{REFERENCIAS}

Alvis, A., C. Velez., H. Villada., M. Rada, Análisis fisicoquímico y morfológico de almidones de ñame yuca y papa y determinación de la viscosidad de las pastas, Información Tecnológica: 19(1),19-28 (2008).

Alvis, A., C. Velez., H. Villada., M. Rada, Composición de ñames frescos cultivados en Colombia y sometidos a freído por inmersión, Información Tecnológica: 19(1), 3-10 (2008).

Amin, A.M., S.A. Ahma., Y.Y.Yin., N.Yahya., N.Ibrahim, Extraction, purification and characterization of durian (Durio zibethinus) seed gum, Food Hydrocolloids: 21, 273-279 (2007).

Andrade, E., J. Maia., R. Streich., F. Marx, Seed Composition of Amazonian Lecythidaceae Species: Part 3 in the Series "Studies of Edible Amazonian Plants", Journal of Food Composition and Analysis: 12, 37-51 (1999).

AOCS Cd 3-25. Official Method Saponification Value, Sampling and Analysis of Commercial Fats and Oils. Copyright The American Oils Chemist's Society. Urbana - Illinois. USA (2003).

AOCS Cd 8-53. Official Method Peroxide Value Acetic Acid (Chloroform Method), Sampling and Analysis of Commercial Fats and Oils. Copyright The American Oils Chemist's Society. Urbana - Illinois. USA (2003).

ASTM D130 - 10. Standard Test Method for Corrosiveness to Copper from Petroleum Products by Copper Strip Test. (2003).

ASTM D 6584. Standard Test Method for Determintion of Total Monoglyceride, Total Diglyceride, Total Triglyceride, and Free and Glycerin by Gas Cromatograpy. (2003).

Belén, D.R., I. López., M. González., M. J. Moreno., C. Medina, Evaluación fisicoquímica de la semilla y del aceite de corozo (Acrocomia aculeata Jac), Grasas y Aceites: 56, 311-316 (2005).

Castro, P., Coello, J., Castillo, L., Opciones para la producción y uso del biodiesel en el Perú, $1^{\circ}$ edición, Soluciones Prácticas ITDG, Lima- Perú, (2007).

Denadai, S. M., y otros cinco autores, In vitro digestibility of globulins from sapucaia (Lecythis pisonis Camb.) nuts by mammalian digestive proteinases, Ciência e Tecnologia de Alimentos, Campinas: 27(3), 535-543 (2007).

FAO / OMS. CODEX Stan 19-1981, Norma del Codex para Grasas y Aceites Comestibles No Regulados por Normas Individuales, Codex Alimentarius Official Standards, Canadá (1999). 
Ferguson, L. R., N. Karunasinghe., S. Zhu, A. H. Wang, Review Selenium and its' role in the maintenance of genomic stability, Mutation Research: 733, 100-110 (2012).

Ferri, T., F. Coccioli., C. De Luca., C.V. Callegari, R. Morabito, Distribution and speciation of selenium in Lecythis ollaría plant, Microchemical journal: 78, 195-203 (2004).

Gerloff, B, Effect of selenium supplementation on dairy cattle, Journal of animal science: 70, 3934-3940 (1992).

Hernández, C., A. Mieres., Z. Niño., S. Pérez, Efecto de la refinación física sobre el aceite de la almendra del corozo (Acrocomia aculeata), Información Tecnológica: 18(4), 59-68 (2007).

Kolachi, N.F., y otros ocho autores, Determination of Selenium Content in Aqueous Extract of Medicinal Plants Used as Herbal Supplement for Cancer Patients, Food and Chemical Toxicology: 48 (12), 3327-3332 (2010).

Lafont, J.J., M.S. Páez., A.A. Portacio, Extracción y Caracterización Fisicoquímica del Aceite de la Semilla (Almendra) del Marañón (Anacardium occidentale L), Información Tecnológica: 22(1), 51-58 (2011).

Lemire M., M. Fillion., F. Jr. Barbosa., J. R. Davée Guimarães., D. Mergler, Elevated Levels Of Selenium In The Typical Diet Of Amazonian Riverside Populations, Science of the Total Environment: 408, 4076-4084 (2010).

Lippi, G., G. Targher., M. Franchini, Vaccination, squalene and anti-squalene antibodies: Facts or fiction?, European Journal of Internal Medicine: 21, 70-73 (2010).

López, C.R y G.I. Montero, Manual de Identificación de Especies Forestales en Bosques Naturales con Manejo Certificable por Comunidades, 128, Instituto Amazónico de Investigaciones Científicas, Bogotá, Colombia (2005).

Müller, D., H. Desel, Acute selenium poisoning by paradise nuts (Lecythis ollaria), Hum Exp Toxicol: 29(5), 431-434 (2010).

Zhang, W. y otros seis autores, Feeding with supplemental squalene enhances the productive performance in boars, Animal Reproduction Science: 104(2-4), 445-449 (2008). 
